\title{
SOME NEW LOWER BOUNDS OF THE FIRST EIGENVALUE ON FINSLER MANIFOLDS
}

\author{
Song-Ting Yin, Qun HE* AND DA-XiaO Zheng
}

\begin{abstract}
We establish some unified lower bounds for the first Neumann and closed eigenvalues of the Finsler-Laplacian on compact Finsler manifolds under the weighted Ricci curvature conditions, which extend some recent theorems on the first eigenvalue of the Riemannian-Laplacian. Moreover, the Lichnerowicz type lower bound for the first Dirichlet eigenvalue of the Finsler-Laplacian is also obtained.
\end{abstract}

\section{Introduction}

For a closed Riemannian $n$-manifold $(M, g)$ with the Ricci curvature satisfying Ric $\geq(n-1) k(k>0)$, Lichnerowicz ([9]) gave the lower bound of the first eigenvalue of Laplacian

$$
\lambda_{1}(M) \geq n k .
$$

When $k=0$, using the gradient estimate, Li-Yau ([8]) obtained

$$
\lambda_{1}(M) \geq \frac{\pi^{2}}{2 d^{2}}
$$

where $d$ is the diameter of $M$. By utilizing the method of barrier function, Zhong-Yang ([27]) further proved that

$$
\lambda_{1}(M) \geq \frac{\pi^{2}}{d^{2}}
$$

Afterwards, Hang-Wang ([7]) showed that $\lambda_{1}(M)>\frac{\pi^{2}}{d^{2}}$ if $n>1$. If $k<0$, it is proved by Li-Yau ([8]) that

$$
\lambda_{1}(M) \geq \frac{1}{(n-1) d^{2}} \exp \left\{-\left[1+\sqrt{-4(n-1)^{2} d^{2} k}\right]\right\} .
$$

2000 Mathematics Subject Classification. Primary 53C60; Secondary 35P15.

Key words and phrases. The first eigenvalue, Finsler-Laplacian, Ricci curvature, $S$ curvature.

This project is supported by NNSFC (11471246), AHNSF (1608085MA03) and NSFHE (KJ2014A257).

* Corresponding author.

Received June 17, 2015; revised September 30, 2015. 
The estimate above was improved by Yang ([22]) to the following

$$
\lambda_{1}(M) \geq \frac{\pi^{2}}{d^{2}} \exp \left\{-\max \{\sqrt{n-1}, \sqrt{2}\} \sqrt{-(n-1) k d^{2}}\right\} .
$$

For the first closed and Neumann eigenvalues, Bakry-Qian ([1]) and ChenWang ([4]) put these two lower bound estimates in the same framework, and gave estimates for the first eigenvalue of very general elliptic symmetric operators, via diameter and Ricci curvature.

It is an important and interesting problem to find a unified lower bound of the first eigenvalue for Laplace operator. For this, Peter Li ([23]) conjectured that for a compact Riemannian manifold $(M, g)$ satisfying Ric $\geq K>0$, the first eigenvalue $\lambda_{1}(M) \geq \frac{\pi^{2}}{d^{2}}+K$. In this direction, Yang ([23]) proved that

$$
\lambda_{1}(M) \geq \frac{\pi^{2}}{d^{2}}+\frac{K}{4} .
$$

Later, this lower bound was improved by Ling ([10]) to

$$
\lambda_{1}(M) \geq \frac{\pi^{2}}{d^{2}}+\frac{31}{100} K
$$

When $K<0$, Yang ([22]) conjectured that $\lambda_{1}(M) \geq \frac{\pi^{2}}{d^{2}}+\frac{K}{2}$. In 2007, Ling ([11]) proved Yang's conjecture. Generally, for a closed Riemannian manifold with Ric $\geq K(K \in R)$, Chen-Wang ([4]) gave that

$$
\lambda_{1}(M) \geq \frac{\pi^{2}}{d^{2}}+\frac{K}{2}
$$

There are many generalizations of the above lower bound estimations. Qian-Zhang-Zhu ([15]) extended them into Alexandrov spaces. Lott-Villani ([12]) generalized Lichnerowicz's estimate to metric measure spaces with curvaturedimension conditions. Wei-Wylie ([20]) and Chu-Hu ([3]) and Futaki-Li-Li ([5]) generalized them to $f$-Laplacian with the $N$-Bakry-Emery Ricci curvature conditions.

As a natural generalization of Riemannian manifolds, Finsler manifolds are differentiable manifolds of which on each tangent space one endows a Minkowskian norm instead of a Euclidean norm. There exists a Finsler-Laplacian, which is a nonlinear elliptic operator, on Finsler manifolds. In recent years, studies on the bound estimations of the first eigenvalue of the Finsler-Laplacian have taken on a new look. In [6] Shen firstly gave the Faber-Krahn type inequality and then further got the Cheng type inequality in [18]. After that, Wu-Xin ([21]) proved Mckean type inequalities, while Chen ([2]) gained the Cheeger type inequality and also improved the Cheng type inequality obtained by Shen. There is still plenty of scope for improvement. Recently, some refinements of the results above were achieved by Yin-He in [24]. As for the first 
Neumann and closed eigenvalues, Wang-Xia ([19]) gave a sharp lower bound estimation on Finsler manifolds with weighted Ricci curvature conditions (see Lemma 2.1 below). In addition, Yin-He-Shen ([24][25][26]) obtained some estimates of the first eigenvalue under the variant weighted Ricci curvature conditions $\left(\operatorname{Ric}_{N} \geq(n-1) k, N \in(n, \infty)\right.$ for $k>0$ or $k<0$ and $\left.\operatorname{Ric}_{\infty} \geq 0\right)$ and further established some rigidity theorems on Finsler manifolds.

But until now, there are no universal explicit estimations of the first eigenvalue on the Finsler manifolds with the weighted Ricci curvature $\operatorname{Ric}_{N} \geq K$ $(N \in[n, \infty], K \in R)$. In this paper we are to answer Li's Conjecture and Yang's Conjecture in the Finsler setting. Here the weighted Ricci curvature and other concepts will be explained in Sec. 1 below.

THEOREM 0.1. Let $(M, F, d \mu)$ be a compact Finsler n-manifold without boundary or with a convex boundary. If the weighted Ricci curvature satisfies $\operatorname{Ric}_{N} \geq K$ for $N \in[n, \infty], K \in R$, then the first closed or Neumann eigenvalue of Finsler-Laplacian

$$
\lambda_{1} \geq 4 s(1-s) \frac{\pi^{2}}{d^{2}}+s K:=C(d, K, s)
$$

where $d$ denotes the diameter of $(M, F)$ and $\forall s \in(0,1)$. In particular,

$$
\lambda_{1} \geq \frac{\pi^{2}}{d^{2}}+\frac{K}{2}
$$

Remark. (i) For any $K$, we give a universal lower bound estimate which is independent of $N$. Moreover, we have

$$
\sup _{s} C(d, K, s)= \begin{cases}0, & K \in\left(-\infty,-\frac{4 \pi^{2}}{d^{2}}\right) \\ \left(\frac{\pi}{d}+\frac{K d}{4 \pi}\right)^{2}, & K \in\left[-\frac{4 \pi^{2}}{d^{2}}, \frac{4 \pi^{2}}{d^{2}}\right] \\ K, & K \in\left(\frac{4 \pi^{2}}{d^{2}}, \frac{(N-1) \pi^{2}}{d^{2}}\right] .\end{cases}
$$

When $N=\infty$ and $K=0$, the estimate $\lambda_{1} \geq \frac{\pi^{2}}{d^{2}}$ is sharp (see [26]). Unfortunately, our estimation gives no information for $K<-\frac{4 \pi^{2}}{d^{2}}$. So we have to give
better estimate when $K<0$.

(ii) Theorem 0.1 generalizes the corresponding results in the Riemannian case and the method of proof is similar (see [3][5]).

(iii) $\lambda_{1}$ is the first Neumann eigenvalue of the Finsler-Laplacian means that $\Delta u=-\lambda_{1} u$ in $M$ with a Neumann boundary condition $\nabla u(x) \in T_{x}(\partial M)$ if $\partial M$ is not empty ([19]). 
We should remark that, in the Finsler setting, the gradient and the Laplacian are both nonlinear operators (see Section 1 below). In general, the following DO NOT hold:

$$
\begin{array}{ll}
\nabla(u+v)=\nabla u+\nabla v, & \nabla(u v)=u \nabla v+v \nabla u, \\
\Delta(u+v)=\Delta u+\Delta v, & \Delta(u v)=u \Delta v+v \Delta u+2\langle\nabla u, \nabla v\rangle .
\end{array}
$$

This creates many difficulties in the calculations. To overcome it, we use the weighted-linear operators, such as the weighted gradient and the weighted Finsler Laplacian. With them, we can convert some nonlinear problems into the linear ones and then do some calculations as in the Riemannian case. It is shown in [6] that the eigenfunction $u$ of the Finsler Laplacian has only the regularity $u \in C^{1, \alpha}(M) \cap C^{\infty}\left(M_{u}\right)$, where $M_{u}:=\{d u \neq 0\}$. The lack of the good regularity forces us to avoid the point $x$ where $d u(x)=0$ and address the issue on $M_{u}$. For example, we can not compute $\Delta u$ in $M \backslash M_{u}$.

It is well known that Riemannian metrics are reversible metrics, but in general, Finsler metrics are not reversible. This means that if $u$ is an eigenfunction of the Finsler-Laplacian, then $-u$ is not necessarily so. In this case, it is an issue to construct an auxiliary function when coping with the gradient estimate.

Finally, and most importantly, we know that Yau's gradient estimate belongs to the linear problem, the gradient and Laplacian, however, are both nonlinear operators in Finsler manifolds. Besides, for any function $f$, if $d f(x)=0$, then $\Delta f$ generally has no definition at the point $x$. Thus we can not use the Finsler Laplacian $\Delta$ to adopt the maximum principle and obtain the gradient estimate, which is the key method in Riemannian geometry. To establish a gradient estimate in the Finsler setting, we have to utilize the maximum principle for the weighted Finsler Laplacian $\Delta^{\nabla u}$ and guarantee that the test function constructed attains its maximum in $M_{u}$.

By the above skill, we may reform the techniques used in the Riemannian case to solve the corresponding problems in Finsler geometry. In view of the Bochner-Weitzenböck formula obtained by Ohta-Sturm ([14]), we then prove the following results.

THEOREM 0.2. Let $(M, F, d \mu)$ be a compact Finsler n-manifold without boundary or with a convex boundary. If the weighted Ricci curvature satisfies $\operatorname{Ric}_{N} \geq K(K<0)$ for $N \in(n, \infty)$, then the first closed or Neumann eigenvalue of Finsler-Laplacian

$$
\lambda_{1} \geq \frac{-C_{N}^{2} \pi^{2} K}{32}\left(e^{C_{N} d \sqrt{-K} / 4}-1\right)^{-2}
$$

where $C_{N}=\max \{\sqrt{N-1}, \sqrt{2}\} . \quad$ In particular, we have the following estimations.

(1) If $-4 \pi^{2} / d^{2} \leq K<0$, then

$$
\lambda_{1} \geq\left(\frac{\pi^{2}}{d^{2}}+\frac{d^{2} K^{2}}{16 \pi^{2}}\right) e^{-C_{N} d \sqrt{-K} / 2} .
$$


(2) If $K \leq-4 \pi^{2} / d^{2}$, then

$$
\lambda_{1} \geq \frac{2 \pi^{2}}{d^{2}} e^{-C_{N} d \sqrt{-K} / 2} .
$$

Remark. (i) From Theorem 0.2, we obtain the universal lower bound

$$
\lambda_{1} \geq \frac{\pi^{2}}{d^{2}} e^{-C_{N} d \sqrt{-K} / 2}
$$

for any compact Finsler manifold with $\operatorname{Ric}_{N} \geq K(K<0)$.

(ii) Theorem 0.2 is a generalization of the results by Yang ([22]) for Riemannian Laplacian and Chu-Hu ([3]) for Riemannian $f$-Laplacian.

For a compact Riemannian $n$-manifold $(M, g)$ with Ricci curvature Ric $\geq$ $(n-1) k>0$, if the non-empty boundary $\partial M$ has nonnegative mean curvature with respect to the outward normal vector, Reilly ([16]) proved that the first Dirichlet eigenvalue of the Laplacian also satisfies $\lambda_{1} \geq n k$.

The second objective of this paper is to extend Reilly type estimate for the first Dirichlet eigenvalue to the Finsler setting. Since the first eigenfunctions are not $C^{\infty}(M)$, we adopt approximation method to build the integral inequality. The key issue is how to choose a suitable convexity of the boundary. On the base of the suitable convexity, we can give a proper estimate on the integral inequality, and then obtain the following theorem.

THEOREM 0.3. Let $(M, F, d \mu)$ be a compact reversible Finsler n-manifold with a mean convex boundary. If $S$ curvature vanishes and the Ricci curvature satisfies Ric $\geq(n-1) k>0$, then the first Dirichlet eigenvalue of Finsler-Laplacian

$$
\lambda_{1} \geq n k \text {. }
$$

The paper is organized as follows. In Section 1, the related fundamentals of Finsler geometry such as Finsler metric, weighted Ricci curvature, gradient vector, Finsler-Laplacian and some lemmas are briefly introduced. The main results will be proved in Sections 2, and 3, respectively.

\section{Preliminaries}

Let $M$ be a smooth $n$-manifold and $\pi: T M \rightarrow M$ be the natural projection from the tangent bundle $T M$. Let $(x, y)$ be a point of $T M$ with $x \in M$, $y \in T_{x} M$, and let $\left(x^{i}, y^{i}\right)$ be the local coordinates on $T M$ with $y=y^{i} \partial / \partial x^{i}$. A Finsler metric on $M$ is a function $F: T M \rightarrow[0,+\infty)$ satisfying the following properties:

(i) Regularity: $F(x, y)$ is smooth in $T M \backslash 0$;

(ii) Positive homogeneity: $F(x, \lambda y)=\lambda F(x, y)$ for $\lambda>0$; 
(iii) Strong convexity: The fundamental quadratic form

$$
g:=g_{i j}(x, y) d x^{i} \otimes d x^{j}, \quad g_{i j}:=\frac{1}{2}\left[F^{2}\right]_{y^{i} y^{j}}
$$

is positively definite.

Let $X=X^{i} \frac{\partial}{\partial x^{i}}$ be a vector field. Then the covariant derivative of $X$ by $v \in T_{x} M$ with reference vector $w \in T_{x} M \backslash 0$ is defined by

$$
D_{v}^{w} X(x):=\left\{v^{j} \frac{\partial X^{i}}{\partial x^{j}}(x)+\Gamma_{j k}^{i}(w) v^{j} X^{k}(x)\right\} \frac{\partial}{\partial x^{i}},
$$

where $\Gamma_{j k}^{i}$ denote the coefficients of the Chern connection.

Given two linearly independent vectors $V, W \in T_{x} M \backslash 0$, the flag curvature is defined by

$$
K(V, W):=\frac{g_{V}\left(R^{V}(V, W) W, V\right)}{g_{V}(V, V) g_{V}(W, W)-g_{V}(V, W)^{2}},
$$

where $R^{V}$ is the Chern curvature

$$
R^{V}(X, Y) Z=D_{X}^{V} D_{Y}^{V} Z-D_{Y}^{V} D_{X}^{V} Z-D_{[X, Y]}^{V} Z .
$$

Then the Ricci curvature for $(M, F)$ is defined as

$$
\operatorname{Ric}(V)=\sum_{i=1}^{n-1} K\left(V, e_{i}\right)
$$

where $e_{1}, \ldots, e_{n-1}, \frac{V}{F(V)}$ form an orthonormal basis of $T_{x} M$ with respect to $g_{V}$.

For a given volume form $d \mu=\sigma(x) d x$ and a vector $V \in T_{x} M \backslash 0$, the distortion of $(M, F, d \mu)$ is defined by

$$
\tau(V):=\ln \frac{\sqrt{\operatorname{det}\left(g_{i j}(V)\right)}}{\sigma} .
$$

To measure the rate of changes of the distortion along geodesics, we define

$$
S(V):=\frac{d}{d t}[\tau(\dot{c}(t))]_{t=0},
$$

where $c(t)$ is the geodesic with $\dot{c}(0)=V . \quad S$ is called the $S$-curvature.

Now we introduce the weighted Ricci curvature on Finsler manifolds, which was defined by Ohta. In the present paper, we reform it as follows: 
Definition $1.1([13])$. Let $(M, F, d \mu)$ be a Finsler $n$-manifold. Given a vector $V \in T_{x} M$, let $\gamma:(-\varepsilon, \varepsilon) \rightarrow M$ be a geodesic with $\gamma(0)=x, \dot{\gamma}(0)=V$. Define

$$
\dot{S}(V):=F^{-2}(V) \frac{d}{d t}[S(\gamma(t), \dot{\gamma}(t))]_{t=0},
$$

where $S(V)$ denotes the $S$-curvature at $(x, V)$.

The weighted Ricci curvature of $(M, F, d \mu)$ is defined by

$$
\left\{\begin{array}{l}
\operatorname{Ric}_{n}(V):= \begin{cases}\operatorname{Ric}(V)+\dot{S}(V), & \text { for } S(V)=0, \\
-\infty, & \text { otherwise, }\end{cases} \\
\operatorname{Ric}_{N}(V):=\operatorname{Ric}(V)+\dot{S}(V)-\frac{S(V)^{2}}{(N-n) F(V)^{2}}, \quad \forall N \in(n, \infty), \\
\operatorname{Ric}_{\infty}(V):=\operatorname{Ric}(V)+\dot{S}(V),
\end{array}\right.
$$

Let $\mathscr{L}: T M \rightarrow T^{*} M$ denote the Legendre transformation, which satisfies $\mathscr{L}(0)=0$ and $\mathscr{L}(\lambda y)=\lambda \mathscr{L}(y)$ for all $\lambda>0$ and $y \in T M \backslash\{0\}$. Then $\mathscr{L}: T M \backslash\{0\}$ $\rightarrow T^{*} M \backslash\{0\}$ is a norm-preserving $C^{\infty}$ diffeomorphism. For a smooth function $u: M \rightarrow R$, the gradient vector of $u$ at $x \in M$ is defined as $\nabla u(x):=\mathscr{L}^{-1}(d u(x)) \in$ $T_{x} M$, which can be written as

$$
\nabla u(x):= \begin{cases}g^{i j}(x, \nabla u) \frac{\partial u}{\partial x^{j}} \frac{\partial}{\partial x^{i}}, & d u(x) \neq 0, \\ 0, & d u(x)=0 .\end{cases}
$$

Set $M_{V}:=\{x \in M \mid V(x) \neq 0\}$ for a vector field $V$ on $M$, and $M_{u}:=M_{\nabla u}$. For a smooth vector field $V$ on $M$ and $x \in M_{V}$, we define $\nabla V(x) \in T_{x}^{*} M \otimes T_{x} M$ by using the covariant derivative as

$$
\nabla V(v):=D_{v}^{V} V(x) \in T_{x} M, \quad v \in T_{x} M .
$$

For a smooth function $u: M \rightarrow R$ and $x \in M_{u}$, We set $\nabla^{2} u(x):=\nabla(\nabla u)(x)$. Let $\left\{e_{a}\right\}_{a=1}^{n}$ be a local orthonormal basis with respect to $g_{\nabla u}$ on $M_{u}$ and put $u_{a b}=g_{\nabla u}\left(D_{e_{a}}^{\nabla u} \nabla u, e_{b}\right)$. Then we have

$$
u_{a b}=u_{b a}, \quad \forall a, b .
$$

Let $V=V^{i} \frac{\partial}{\partial x^{i}}$ be a smooth vector field on $M$. The divergence of $V$ with respect to an arbitrary volume form $d \mu$ is defined by

$$
\operatorname{div} V:=\sum_{i=1}^{n}\left(\frac{\partial V^{i}}{\partial x^{i}}+V^{i} \frac{\partial \Phi}{\partial x^{i}}\right)
$$

where $d \mu=e^{\Phi} d x$. Then the Finsler-Laplacian of $u$ can be defined by

$$
\Delta u:=\operatorname{div}(\nabla u)
$$


NEW LOWER BOUNDS OF THE FIRST EIGENVALUE ON FINSLER MANIFOLDS 325

Given a vector field $V$ such that $V \neq 0$ on $M_{u}$, the weighted gradient vector and the weighted Laplacian on the weighted Riemannian manifold $\left(M, g_{V}\right)$ are defined by

$$
\nabla^{V} u:=\left\{\begin{array}{ll}
g^{i j}(V) \frac{\partial u}{\partial x^{j}} \frac{\partial}{\partial x^{i}}, & \text { in } M_{u}, \\
0, & \text { in } M \backslash M_{u},
\end{array} \quad \Delta^{V} u:=\operatorname{div}\left(\nabla^{V} u\right) .\right.
$$

We note that $\nabla^{\nabla u} u=\nabla u, \Delta^{\nabla u} u=\Delta u$.

Let $(M, F)$ be a Finsler manifold with boundary $\partial M$ and $v$ be the normal vector that points outward $M$. Here a normal vector $v$ at $x \in \partial M$ means that for any $w \in T_{x}(\partial M), g_{v}(v, w)=0$. There are exactly two normal vectors $v, v^{\prime} \in T_{x} M$. In general, $v^{\prime} \neq-v$. The normal curvature $\Lambda_{v}(V)$ at $x \in \partial M$ in direction $V \in T_{x}(\partial M)$ is defined by $([17])$

$$
\Lambda_{v}(V):=g_{v}\left(v,\left.D_{\dot{\gamma}}^{\dot{\gamma}}\right|_{x}\right),
$$

where $\gamma$ is the unique local geodesic for the Finsler structure $F_{\partial M}$ on $\partial M$ induced by $F$ with the initial data $\gamma(0)=x$ and $\dot{\gamma}(0)=V . M$ is said to have convex boundary if for any $x \in \partial M$, the normal curvature $\Lambda$ at $x$ is non-positive in any directions $V \in T_{x}(\partial M)$.

Definition 1.2 ([26]). Let $\Omega \subset M$ be a smooth domain of a reversible Finsler manifold $(M, F)$. The boundary $\partial \Omega$ is called mean convex if there exists (and then for all) a $C^{2}$ function $\phi$ satisfying

$$
\begin{cases}\phi_{i}(x)=0, & x \in U \cap \partial \Omega \\ \phi_{i}(x)>0, & x \in U \cap \Omega \\ d \phi_{i}(x) \neq 0, & x \in U \cap \partial \Omega\end{cases}
$$

where $U$ is a neighborhood of $x \in \partial \Omega$, such that $F(\nabla \phi) H=\sum_{a=1}^{n-1} \nabla^{2} \phi\left(e_{a}, e_{a}\right) \leq 0$. Here $\left\{e_{a}\right\}_{a=1}^{n}$ is a $g_{\nabla \phi}$-orthogonal frame field with $e_{n}=\frac{\nabla \phi}{F(\nabla \phi)}$.

Lemma $1.3([14])$. Let $(M, F)$ be a Finsler n-manifold. Given $u \in C^{\infty}(M)$, we have

$$
\Delta^{\nabla u}\left(\frac{F(\nabla u)^{2}}{2}\right)-D(\Delta u)(\nabla u)=|\nabla u|^{2} \operatorname{Ric}_{\infty}(\nabla u)+\left|\nabla^{2} u\right|_{H S(\nabla u)}^{2}
$$

point-wise on $M_{u}$. Here $\left|\nabla^{2} u\right|_{H S(\nabla u)}^{2}$ stands for the Hilbert-Schmidt norm with respect to $g_{\nabla u}$.

Lemma 1.4 ([21]). Let $(M, F)$ be a Finsler n-manifold and $u: M \rightarrow R$ be a smooth function. Then on $M_{u}$ we have

$$
\Delta u=\operatorname{tr}_{g_{\nabla u}}\left(\nabla^{2} u\right)-S(\nabla u)=\sum_{a} u_{a a}-S(\nabla u),
$$

where $u_{a a}=g_{\nabla u}\left(\nabla^{2} u\left(e_{a}\right), e_{a}\right)$ and $\left\{e_{a}\right\}_{a=1}^{n}$ is a local $g_{\nabla u}$-orthonormal basis on $M_{u}$. 


\section{The first Neumann and closed eigenvalue}

Before the proof of our main results, we introduce the following

Lemma 2.1 ([19]). Let $\left(M^{n}, F, m\right)$ be an n-dimensional compact Finsler measure space, equipped with a Finsler structure $F$ and a smooth measure $m$, without boundary or with a convex boundary. Assume that $\operatorname{Ric}_{N} \geq K$ for some real numbers $N \in[n, \infty]$ and $K \in R$. Let $\lambda_{1}$ be the first Neumann eigenvalue of the Finsler-Laplacian if $\partial M$ is not empty. Then

$$
\lambda_{1} \geq \lambda_{1}(K, N, d)
$$

where $d$ is the diameter of $M, \lambda_{1}(K, N, d)$ represents the first eigenvalue of the 1-dimensional problem

$$
v^{\prime \prime}-T(t) v^{\prime}=-\lambda_{1}(K, N, d) v \quad \text { in }\left(-\frac{d}{2}, \frac{d}{2}\right), \quad v^{\prime}\left(-\frac{d}{2}\right)=v^{\prime}\left(\frac{d}{2}\right)=0
$$

where $T(t)$ is defined by

$$
T(t)= \begin{cases}\sqrt{(N-1) K} \tan \left(\sqrt{\frac{K}{N-1}} t\right), & K>0,1<N<\infty \\ -\sqrt{-(N-1) K} \tanh \left(\sqrt{\left.-\frac{K}{N-1} t\right),}\right. & K<0,1<N<\infty \\ 0, & K=0,1<N<\infty \\ K t, & N=\infty\end{cases}
$$

Using the above Lemma and similar arguments as in the Riemaniann case, it is easy to prove

THEOREM 2.2. Let $(M, F, d \mu)$ be a compact Finsler n-manifold without boundary or with a convex boundary. If the weighted Ricci curvature satisfies $\operatorname{Ric}_{N} \geq K$ for $N \in[n, \infty], K \in R$, then the first closed or Neumann eigenvalue of Finsler-Laplacian

$$
\lambda_{1} \geq 4 s(1-s) \frac{\pi^{2}}{d^{2}}+s K:=C(d, K, s)
$$

where $d$ denotes the diameter of $(M, F)$ and $\forall s \in(0,1)$. In particular,

$$
\lambda_{1} \geq \frac{\pi^{2}}{d^{2}}+\frac{K}{2}
$$

Proof. Differentiating both sides of (2.1), we have

$$
v^{\prime \prime \prime}-T^{\prime} v^{\prime}-T v^{\prime \prime}=-\lambda_{1}(K, N, d) v^{\prime} .
$$


Multiplying (2.3) by $\left(v^{\prime}\right)^{a}$ for $a>0$ and integrating it over $\left(-\frac{d}{2}, \frac{d}{2}\right)$ yields

$$
\begin{gathered}
\int_{-d / 2}^{d / 2} v^{\prime \prime \prime}\left(v^{\prime}\right)^{a} d t-\int_{-d / 2}^{d / 2} T^{\prime}\left(v^{\prime}\right)^{1+a} d t-\int_{-d / 2}^{d / 2} T v^{\prime \prime}\left(v^{\prime}\right)^{a} d t \\
\quad=-\lambda_{1}(K, N, d) \int_{-d / 2}^{d / 2}\left(v^{\prime}\right)^{1+a} d t .
\end{gathered}
$$

Notice that $v^{\prime}>0$ in $\left(-\frac{d}{2}, \frac{d}{2}\right)$ (see [19] for details) and $v^{\prime}\left(-\frac{d}{2}\right)=v^{\prime}\left(\frac{d}{2}\right)=0$,
we get

$$
\begin{aligned}
\int_{-d / 2}^{d / 2} v^{\prime \prime \prime}\left(v^{\prime}\right)^{a} d t & =-a \int_{-d / 2}^{d / 2}\left(v^{\prime \prime}\right)^{2}\left(v^{\prime}\right)^{a-1} d t \\
& =-\frac{4 a}{(1+a)^{2}} \int_{-d / 2}^{d / 2}\left(\left(\left(v^{\prime}\right)^{(a+1) / 2}\right)^{\prime}\right)^{2} d t
\end{aligned}
$$

In addition,

(2.6) $-\int_{-d / 2}^{d / 2} T v^{\prime \prime}\left(v^{\prime}\right)^{a} d t=-\frac{1}{1+a} \int_{-d / 2}^{d / 2} T d\left(v^{\prime}\right)^{a+1}=\frac{1}{1+a} \int_{-d / 2}^{d / 2} T^{\prime}\left(v^{\prime}\right)^{a+1} d t$.

Substituting (2.5) and (2.6) into (2.4), one obtains

$$
\frac{4 a}{(1+a)^{2}} \int_{-d / 2}^{d / 2}\left(\left(\left(v^{\prime}\right)^{(a+1) / 2}\right)^{\prime}\right)^{2} d t=\int_{-d / 2}^{d / 2}\left(\lambda_{1}(K, N, d)-\frac{a}{a+1} T^{\prime}\right)\left(v^{\prime}\right)^{a+1} d t .
$$

Since $v^{\prime}\left(-\frac{d}{2}\right)=v^{\prime}\left(\frac{d}{2}\right)=0$, by using Wirtinger's inequality

$$
\int_{-l}^{l} \phi^{2}(x) d x \leq\left(\frac{2 l}{\pi}\right)^{2} \int_{-l}^{l}\left(\phi^{\prime}\right)^{2}(x) d x, \quad \forall \phi \in C^{1}[-l, l], \phi( \pm l)=0,
$$

we achieve that

$$
\begin{aligned}
\frac{4 a}{(1+a)^{2}} \frac{\pi^{2}}{d^{2}} \int_{-d / 2}^{d / 2}\left(v^{\prime}\right)^{a+1} d t & \leq \int_{-d / 2}^{d / 2}\left(\lambda_{1}(K, N, d)-\frac{a}{a+1} T^{\prime}\right)\left(v^{\prime}\right)^{a+1} d t \\
& \leq\left(\lambda_{1}(K, N, d)-\frac{a}{a+1} \min _{t \in[-d / 2, d / 2]} T^{\prime}\right) \int_{-d / 2}^{d / 2}\left(v^{\prime}\right)^{a+1} d t
\end{aligned}
$$

It is easy to check that $T^{\prime}=K+\frac{T^{2}}{N-1}$. Hence,

$$
\lambda_{1}(K, N, d) \geq \frac{4 a}{(a+1)^{2}} \frac{\pi^{2}}{d^{2}}+\frac{a}{a+1} K
$$


Taking $s=\frac{a}{a+1}$ and using Lemma 2.1, we conclude

$$
\lambda_{1} \geq 4 s(1-s) \frac{\pi^{2}}{d^{2}}+s K:=C(d, K, s) .
$$

View the right side of above formula as a quadratic polynomial on $s$, and note that $d \leq \frac{\sqrt{N-1} \pi}{\sqrt{K}}, K>0$ (see [13] for details), then it is not hard to get $\sup _{s} C(d, K, s)$ in the remark.

THEOREM 2.3. Let $(M, F, d \mu)$ be a compact Finsler n-manifold without boundary or with a convex boundary. If the weighted Ricci curvature satisfies $\operatorname{Ric}_{N} \geq K(K<0)$ for $N \in(n, \infty)$, then the first closed or Neumann eigenvalue of Finsler-Laplacian

$$
\lambda_{1} \geq \frac{-C_{N}^{2} \pi^{2} K}{32}\left(e^{C_{N} d \sqrt{-K} / 4}-1\right)^{-2},
$$

where $C_{N}=\max \{\sqrt{N-1}, \sqrt{2}\} . \quad$ In particular, we have the following estimations.

(1) If $-4 \pi^{2} / d^{2} \leq K<0$, then

$$
\lambda_{1} \geq\left(\frac{\pi^{2}}{d^{2}}+\frac{d^{2} K^{2}}{16 \pi^{2}}\right) e^{-C_{N} d \sqrt{-K} / 2} .
$$

(2) If $K \leq-4 \pi^{2} / d^{2}$, then

$$
\lambda_{1} \geq \frac{2 \pi^{2}}{d^{2}} e^{-C_{N} d \sqrt{-K} / 2} .
$$

Remark. Before the proof, we give some explication. Since gradient and Laplacian are nonlinear operators in Finsler manifolds, the calculations are not so easy as in the Riemannian case. For instance, $\nabla(u+v) \neq \nabla u+\nabla v$ and $\nabla(c u) \neq c \nabla u$ for $c \in \mathbf{R}$ in general. We construct the weighted operators $\nabla^{\nabla u}$ and $\Delta^{\nabla u}$ with the reference direction $\nabla u$. This is because these weighted operators are linear operators and moreover in $M_{u}$ we have $\nabla^{\nabla u} u=\nabla u, \Delta^{\nabla u} u=\Delta u$. We should also point out that $\Delta^{\nabla u}$ satisfies the maximal principle on $M_{u}$ but have no sense on $M \backslash M_{u}$. Therefore, with the weighted operators at hand, we can use the technique similar to the Riemannian case to handle the problems on $M_{u}$ but need to give additional discussion on $M \backslash M_{u}$.

Proof. We firstly consider the case that $\partial M=\emptyset$. Let $u$ be a first eigenfunction on $(M, F)$ corresponding to the first eigenvalue $\lambda_{1}$. Since $\int_{M} u d \mu=$ $-\frac{1}{\lambda_{1}} \int_{M} \Delta u d \mu=0$ and noting that $-u$ is not necessarily the first eigenfunction on $(M, F)$, we assume that

$$
1=\sup u>\inf u=-k \geq-1 \quad(\text { resp. } 1 \geq k=\sup u>\inf u=-1), \quad 0<k \leq 1 .
$$


For small $\varepsilon>0$, let

$$
v=\frac{u-\frac{1}{2}(1-k)}{\frac{1}{2}(1+k)(1+\varepsilon)} \quad\left(\text { resp. } v=\frac{u+\frac{1}{2}(1-k)}{\frac{1}{2}(1+k)(1+\varepsilon)}\right) .
$$
Clearly, $d v=\frac{2}{(1+k)(1+\varepsilon)} d u$. Thus by the property of Legendre transform we
have

$$
\nabla v=\nabla^{\nabla} u v=\frac{2}{(1+k)(1+\varepsilon)} \nabla u
$$

under which

$$
\begin{cases}\Delta v=-\lambda_{1}\left(v \pm a_{\varepsilon}\right), & a_{\varepsilon}=\frac{1-k}{(1+k)(1+\varepsilon)}, \\ \sup v=\frac{1}{1+\varepsilon}, & \text { inf } v=-\frac{1}{1+\varepsilon} .\end{cases}
$$

Let $v=\sin \theta$, then $d v=\cos \theta d \theta$ and

$$
-\frac{1}{1+\varepsilon} \leq \sin \theta \leq \frac{1}{1+\varepsilon}, \quad \frac{|\nabla v|^{2}}{1-v^{2}}=|\nabla \theta|^{2} .
$$

We note that $\theta \in\left[-\frac{\pi}{2}+\delta, \frac{\pi}{2}-\delta\right]$, where $\delta$ satisfies $\sin \left(\frac{\pi}{2}-\delta\right)=\frac{1}{1+\varepsilon}$, and that $\nabla \theta=\nabla^{\nabla v} \theta$ since $\cos \theta>0$. Set

$$
U(\theta):=\max _{x \in M, \theta(x)=\theta}|\nabla \theta|^{2}=\max _{x \in M, \theta(x)=\theta} \frac{|\nabla v|^{2}}{1-v^{2}}, \quad \theta \in\left[-\frac{\pi}{2}+\delta, \frac{\pi}{2}-\delta\right] .
$$

Here and from now on, we use $|\nabla \theta|,|\nabla v|$ to denote $F(\nabla \theta), F(\nabla v)$. Then $U(\theta)$ is continuous on $\left[-\frac{\pi}{2}+\delta, \frac{\pi}{2}-\delta\right]$. Moreover, for any $\theta_{0} \in\left(-\frac{\pi}{2}+\delta, \frac{\pi}{2}-\delta\right)$, there exists a point $x_{0} \in M$ such that $\theta\left(x_{0}\right)=\theta_{0}$ and $U\left(\theta_{0}\right)=|\nabla \theta|^{2}\left(x_{0}\right)$.

Now we claim that

$$
|\nabla \theta| \leq \sqrt{\lambda_{1}\left(1+a_{\varepsilon}\right)}+b \sqrt{-K} \cos \theta, \quad \theta \in\left[-\frac{\pi}{2}+\delta, \frac{\pi}{2}-\delta\right],
$$

where $b=\frac{1}{2} \max \{\sqrt{N-1}, \sqrt{2}\}:=\frac{1}{2} C_{N}$. If it is not true, one would take $x_{0}$ attaining

$$
A:=\max _{x \in M}\{|\nabla \theta(x)|-b \sqrt{-K} \cos \theta(x)\} .
$$

and put $\theta_{0}:=\theta\left(x_{0}\right)$. Clearly,

$$
A>\sqrt{\lambda_{1}\left(1+a_{\varepsilon}\right)} .
$$


If $\sqrt{U(\theta)}-b \sqrt{-K} \cos \theta$ attains its maximum at $x_{0} \in M \backslash M_{u}$, then

$$
\begin{aligned}
\sqrt{U(\theta)}-b \sqrt{-K} \cos \theta & \leq \sqrt{U\left(\theta_{0}\right)}-b \sqrt{-K} \cos \theta_{0} \\
& =-b \sqrt{-K} \cos \theta_{0} \leq \sqrt{\lambda_{1}\left(1+a_{\varepsilon}\right)} .
\end{aligned}
$$

The claim holds undoubtedly. Next, we suppose that $\sqrt{U(\theta)}-b \sqrt{-K} \cos \theta$ attains its maximum at $x_{0} \in M_{u}$. Put

$$
G(x):=\left\{\frac{|\nabla v|^{2}}{1-v^{2}}-(A+b \sqrt{-K} \cos \theta)^{2}\right\} \cos ^{2} \theta .
$$

By the definitions of $U(\theta)$ and $A$, we have at $x_{0}$ that

$$
\left\{\begin{array}{l}
G\left(x_{0}\right)=0, \\
\nabla^{\nabla u} G\left(x_{0}\right)=0, \\
\Delta^{\nabla u} G\left(x_{0}\right) \leq 0 .
\end{array}\right.
$$

Let $e_{1}, e_{2}, \ldots, e_{n}=\frac{\nabla u}{|\nabla u|}=\frac{\nabla v}{|\nabla v|}$ be a local orthonormal basis with respect to $g_{\nabla u}$ on $M_{u}$. Then by simple computations on $\nabla^{\nabla u} G\left(x_{0}\right)=0$ we have

$$
\left\{\begin{aligned}
v_{a n} & =0, \quad a \neq n \\
v_{n n} & =-(A+b \sqrt{-K} \cos \theta)(A+2 b \sqrt{-K} \cos \theta) \sin \theta \\
& =\frac{-v|\nabla v|^{2}}{1-v^{2}}-b \sqrt{-K} v|\nabla v|
\end{aligned}\right.
$$

Furthermore, a straightforward calculation yields

$$
\begin{aligned}
0 \geq \Delta^{\nabla u} G\left(x_{0}\right)= & \cos ^{2} \theta \Delta^{\nabla u}\left(\frac{|\nabla v|^{2}}{1-v^{2}}-(A+b \sqrt{-K} \cos \theta)^{2}\right) \\
& +\left(\frac{|\nabla v|^{2}}{1-v^{2}}-(A+b \sqrt{-K} \cos \theta)^{2}\right) \Delta^{\nabla u} \cos ^{2} \theta \\
& +2 g_{\nabla u}\left(\nabla^{\nabla u}\left(\frac{|\nabla v|^{2}}{1-v^{2}}-(A+b \sqrt{-K} \cos \theta)^{2}\right), \nabla^{\nabla u} \cos ^{2} \theta\right) \\
= & \cos ^{2} \theta \Delta^{\nabla u}\left(\frac{|\nabla v|^{2}}{1-v^{2}}-(A+b \sqrt{-K} \cos \theta)^{2}\right)+0+0 \\
= & \cos ^{2} \theta \Delta^{\nabla u}\left(\frac{|\nabla v|^{2}}{1-v^{2}}\right)-\cos ^{2} \theta \Delta^{\nabla u}\left((A+b \sqrt{-K} \cos \theta)^{2}\right) .
\end{aligned}
$$


On the other hand,

$$
\begin{aligned}
\Delta^{\nabla u}\left(\frac{|\nabla v|^{2}}{1-v^{2}}\right)= & \frac{\Delta^{\nabla u}\left(|\nabla v|^{2}\right)}{1-v^{2}}+|\nabla v|^{2} \Delta^{\nabla u}\left(\frac{1}{1-v^{2}}\right) \\
& +2 g_{\nabla u}\left(\nabla^{\nabla u}\left(|\nabla v|^{2}\right), \nabla^{\nabla u}\left(\frac{1}{1-v^{2}}\right)\right) \\
:= & A+B+C
\end{aligned}
$$

where

$$
\begin{aligned}
A & =\frac{\Delta^{\nabla u}\left(|\nabla v|^{2}\right)}{1-v^{2}} \\
B & =|\nabla v|^{2} \Delta^{\nabla u}\left(\frac{1}{1-v^{2}}\right)=|\nabla v|^{2} \operatorname{div}\left(\nabla^{\nabla u}\left(\frac{1}{1-v^{2}}\right)\right) \\
& =|\nabla v|^{2}\left\{\frac{2 v}{\left(1-v^{2}\right)^{2}} \operatorname{div}\left(\nabla^{\nabla u} v\right)+2 g_{\nabla u}\left(\nabla^{\nabla u} v, \nabla^{\nabla u}\left(\frac{v}{\left(1-v^{2}\right)^{2}}\right)\right)\right\} \\
& =|\nabla v|^{2}\left\{\frac{2 v}{\left(1-v^{2}\right)^{2}} \Delta v+\frac{2|\nabla v|^{2}}{\left(1-v^{2}\right)^{2}}+\frac{8 v^{2}|\nabla v|^{2}}{\left(1-v^{2}\right)^{3}}\right\} \\
C & =2 g_{\nabla u}\left(\nabla^{\nabla u}\left(|\nabla v|^{2}\right), \nabla^{\nabla u}\left(\frac{1}{1-v^{2}}\right)\right) \\
& =\frac{8 v|\nabla v|^{2}}{\left(1-v^{2}\right)^{2}} v_{n n}=-\frac{8 v^{2}|\nabla v|^{4}}{\left(1-v^{2}\right)^{3}}-\frac{8 b \sqrt{-K} v^{2}|\nabla v|^{3}}{\left(1-v^{2}\right)^{2}}
\end{aligned}
$$

By Lemma 1.3 and the conditions of Theorem 2.3, we get

$$
\begin{aligned}
\Delta^{\nabla u}\left(|\nabla v|^{2}\right)= & 2|\nabla v|^{2} \operatorname{Ric}_{\infty}(\nabla v)+2 D(\Delta v)(\nabla v)+2\left|\nabla^{2} v\right|_{H S(\nabla v)}^{2} \\
= & 2|\nabla v|^{2} \operatorname{Ric}_{\infty}(\nabla v)+2 D\left(-\lambda_{1}\left(v \pm a_{\varepsilon}\right)\right)(\nabla v)+2 \sum_{a b} v_{a b}^{2} \\
\geq & 2|\nabla v|^{2} \operatorname{Ric}_{\infty}(\nabla v)-2 \lambda_{1}|\nabla v|^{2}+2\left(v_{n n}^{2}+\sum_{a=1}^{n-1} v_{a a}^{2}\right) \\
\geq & 2|\nabla v|^{2} \operatorname{Ric}_{\infty}(\nabla v)-2 \lambda_{1}|\nabla v|^{2}+2 v_{n n}^{2}+\frac{2}{n-1}\left(\sum_{a=1}^{n-1} v_{a a}\right)^{2} \\
= & 2|\nabla v|^{2} \operatorname{Ric}_{\infty}(\nabla v)-2 \lambda_{1}|\nabla v|^{2}+2 v_{n n}^{2} \\
& +\frac{2}{n-1}\left(\Delta v-v_{n n}+S(\nabla v)\right)^{2}
\end{aligned}
$$




$$
\begin{aligned}
\geq & 2|\nabla v|^{2} \operatorname{Ric}_{\infty}(\nabla v)-2 \lambda_{1}|\nabla v|^{2}+2 v_{n n}^{2} \\
& +\frac{2}{N-1}\left(\Delta v-v_{n n}\right)^{2}-\frac{2 S(\nabla v)^{2}}{N-n} \\
= & 2|\nabla v|^{2} \operatorname{Ric}_{N}(\nabla v)-2 \lambda_{1}|\nabla v|^{2}+2 v_{n n}^{2}+\frac{2}{N-1}\left(\Delta v-v_{n n}\right)^{2} \\
\geq & -2\left(\lambda_{1}-K\right)|\nabla v|^{2}+2 v_{n n}^{2}+\frac{2}{N-1}\left(\Delta v-v_{n n}\right)^{2}
\end{aligned}
$$

Therefore we have that

$$
\begin{aligned}
\cos ^{2} \theta \Delta^{\nabla u}\left(\frac{|\nabla v|^{2}}{1-v^{2}}\right) \geq & -2\left(\lambda_{1}-K\right)|\nabla v|^{2}+2 v_{n n}^{2}+\frac{2}{N-1}\left(\Delta v-v_{n n}\right)^{2} \\
& +2|\nabla v|^{2}\left\{\frac{v \Delta v+|\nabla v|^{2}}{\left(1-v^{2}\right)}\right\}-\frac{8 b \sqrt{-K} v^{2}|\nabla v|^{3}}{1-v^{2}}
\end{aligned}
$$

at $x_{0}$. In addition,

$$
\begin{aligned}
&-\cos ^{2} \theta \Delta^{\nabla u}((A\left.+b \sqrt{-K} \cos \theta)^{2}\right) \\
&=-\cos ^{2} \theta[ 2(A+b \sqrt{-K} \cos \theta) \Delta^{\nabla u}(A+b \sqrt{-K} \cos \theta) \\
&\left.+2 g_{\nabla u}\left(b \sqrt{-K} \nabla^{\nabla u} \cos \theta, b \sqrt{-K} \nabla^{\nabla u} \cos \theta\right)\right] \\
&=-\cos ^{2} \theta[ 2 b \sqrt{-K}(A+b \sqrt{-K} \cos \theta) \\
&\left.\times\left(\frac{\lambda_{1} \sin \theta\left(\sin \theta \pm a_{\varepsilon}\right)}{\cos \theta}-\frac{|\nabla \theta|^{2}}{\cos \theta}\right)-2 b^{2} K \sin ^{2} \theta|\nabla \theta|^{2}\right] \\
&=2 b \sqrt{-K}(A+b \sqrt{-K} \cos \theta)\left(-\lambda_{1} \sin \theta \cos \theta\left(\sin \theta \pm a_{\varepsilon}\right)+\cos \theta|\nabla \theta|^{2}\right) \\
&+2 b^{2} K \sin ^{2} \theta \cos ^{2} \theta|\nabla \theta|^{2} .
\end{aligned}
$$

Setting $y:=A+b \sqrt{-K} \cos \theta$ and substituting (2.15) (2.16) into (2.13), we have

$$
\begin{aligned}
y^{2} \leq & \lambda_{1}\left(1+a_{\varepsilon}\right)-K \cos ^{2} \theta+b y \sqrt{-K} \cos \theta-2 A b \sqrt{-K} \cos ^{3} \theta \\
& +2 b^{2} K \cos ^{4} \theta+b \sqrt{-K} \cos \theta\left|\frac{\lambda_{1}\left(1+a_{\varepsilon}\right)}{y} \sin \theta\right| \\
& -\frac{1}{N-1}\left[\frac{\lambda_{1}\left(-\sin \theta \mp a_{\varepsilon}\right)}{y}+A \sin \theta+2 b \sqrt{-K} \cos \theta \sin \theta\right]^{2} .
\end{aligned}
$$

Notice that

$$
\left|\frac{\lambda_{1}\left(1+a_{\varepsilon}\right)}{y} \sin \theta\right| \leq \sqrt{\lambda_{1}\left(1+a_{\varepsilon}\right)} \leq A
$$


and

$$
\begin{aligned}
& -\frac{4}{N-1}\left[\frac{\lambda_{1}\left(-\sin \theta \mp a_{\varepsilon}\right)}{y}+A \sin \theta\right] b \sqrt{-K} \cos \theta \sin \theta \\
& \quad=-\frac{4}{N-1}\left[\frac{\lambda_{1}\left(-\sin \theta \mp a_{\varepsilon}\right)}{y} \sin \theta+A\right] b \sqrt{-K} \cos \theta+\frac{4}{N-1} A b \sqrt{-K} \cos ^{3} \theta \\
& \quad \leq \frac{4}{N-1} A b \sqrt{-K} \cos ^{3} \theta,
\end{aligned}
$$

one gets from (2.17) that

$$
\begin{aligned}
(y- & \left.\frac{1}{2} b \sqrt{-K} \cos \theta\right)^{2} \\
\leq & \left(\sqrt{\lambda_{1}\left(1+a_{\varepsilon}\right)}+\frac{1}{2} b \sqrt{-K} \cos \theta\right)^{2}-K\left(1-\frac{4 b^{2}}{N-1}\right) \cos ^{2} \theta \\
& +\left(\frac{4}{N-1}-2\right) A b \sqrt{-K} \cos ^{3} \theta-\left(\frac{4}{N-1}-2\right) b^{2} K \cos ^{4} \theta .
\end{aligned}
$$

If $N \geq 3$, by choosing $b=\frac{1}{2} \sqrt{N-1}$, then (2.18) implies

$$
y \leq \sqrt{\lambda_{1}\left(1+a_{\varepsilon}\right)}+b \sqrt{-K} \cos \theta
$$

which contradicts (2.11). If $N \leq 3$, then

$$
\begin{aligned}
& -\frac{1}{N-1}\left[\frac{\lambda_{1}\left(-\sin \theta \mp a_{\varepsilon}\right)}{y}+A \sin \theta+2 b \sqrt{-K} \cos \theta \sin \theta\right]^{2} \\
& \leq-\frac{1}{2}\left[\frac{\lambda_{1}\left(-\sin \theta \mp a_{\varepsilon}\right)}{y}+A \sin \theta+2 b \sqrt{-K} \cos \theta \sin \theta\right]^{2} .
\end{aligned}
$$

By a similar argument, we can also draw the conclusion. Thus we have proved that

$$
|\nabla \theta| \leq \sqrt{\lambda_{1}\left(1+a_{\varepsilon}\right)}+b \sqrt{-K} \cos \theta, \quad \theta \in\left[-\frac{\pi}{2}+\delta, \frac{\pi}{2}-\delta\right],
$$

where $b=\frac{1}{2} \max \{\sqrt{N-1}, \sqrt{2}\}:=\frac{1}{2} C_{N}$.

Let $p, q \in M$ be two points such that $\theta(p)=-\frac{\pi}{2}+\delta, \theta(q)=\frac{\pi}{2}-\delta$. Let $\gamma$ be a shortest geodesic from $p$ to $q$. Denote by $T$ the tangent vector of $\gamma$. Then

$$
\begin{aligned}
|\nabla \theta| & =\frac{|\nabla v|}{\cos \theta}=\frac{F(\nabla v)}{\cos \theta} \geq \frac{g_{\nabla u}\left(\nabla v, \frac{T}{F(T)}\right)}{\cos \theta} \\
& =\frac{T v}{F(T) \cos \theta}=\frac{\frac{d v}{d s}}{F(T) \cos \theta}=\frac{\frac{d \theta}{d s}}{F(T)} .
\end{aligned}
$$


We restrict $s$ to $\gamma$ with $\frac{d \theta}{d s} \geq 0$. Then the following integration $\int d \theta$ still covers whole $\left(-\frac{\pi}{2}+\delta, \frac{\pi}{2}-\delta\right)$. Therefore, from (2.19), one gets

$$
\begin{aligned}
d & \geq \int_{\gamma} F(T) d s \geq \int_{-\pi / 2+\delta}^{\pi / 2-\delta} \frac{d \theta}{\sqrt{\lambda_{1}\left(1+a_{\varepsilon}\right)}+\frac{1}{2} C_{N} \sqrt{-K} \cos \theta} \\
& \geq \int_{-\pi / 2+\delta}^{\pi / 2-\delta} \frac{d \theta}{\sqrt{\lambda_{1}\left(1+a_{\varepsilon}\right)}+\frac{1}{2} C_{N} \sqrt{-K}\left(\frac{\pi}{2}-|\theta|\right)} \\
& =\frac{4}{C_{N} \sqrt{-K}} \log \frac{\sqrt{\lambda_{1}\left(1+a_{\varepsilon}\right)}+\frac{\pi}{4} C_{N} \sqrt{-K}}{\sqrt{\lambda_{1}\left(1+a_{\varepsilon}\right)}+\frac{1}{2} C_{N} \delta} .
\end{aligned}
$$

Let $\varepsilon \rightarrow 0$, then $\delta \rightarrow 0$ too. Thus we obtain

$$
\lambda_{1} \geq \frac{C_{N}^{2} \pi^{2}(-K)}{32}\left[\exp \left(\frac{C_{N}}{4} d \sqrt{-K}\right)-1\right]^{-2} .
$$

By using the inequality $\left(e^{x}-1\right)^{2} \leq e^{2 x}-1, \forall x \geq 0$, we further have

$$
\begin{aligned}
\lambda_{1} & \geq \frac{C_{N}^{2} \pi^{2}(-K)}{32}\left[\exp \left(\frac{C_{N}}{4} d \sqrt{-K}\right)-1\right]^{-2} \\
& \geq \frac{-K}{2}\left[\exp \left(\frac{C_{N}}{4} d \sqrt{-K}\right)-1\right]^{-2} \\
& \geq \frac{-K}{2}\left[\exp \left(\frac{C_{N}}{2} d \sqrt{-K}\right)-1\right]^{-1}
\end{aligned}
$$

which implies

$$
\frac{K}{2} \geq \lambda_{1}\left(1-\exp \left(\frac{C_{N}}{2} d \sqrt{-K}\right)\right) .
$$

From the remark below Theorem 0.1 , we know that $\lambda_{1} \geq \frac{\pi^{2}}{d^{2}}+\frac{K}{2}+\frac{d^{2} K^{2}}{16 \pi^{2}}$ for $K \in\left[\frac{-4 \pi^{2}}{d^{2}}, 0\right]$. This together with (2.20) gives (2.9). If $K \leq \frac{-4 \pi^{2}}{d^{2}}$, then from (2.20) we have

$$
\frac{-2 \pi^{2}}{d^{2}} \geq \lambda_{1}\left(1-\exp \left(\frac{C_{N}}{2} d \sqrt{-K}\right)\right),
$$

which implies (2.10).

Now we consider the case that $\partial M \neq \emptyset$. Let $u$ be a first Neumann eigenfunction corresponding to the first eigenvalue $\lambda_{1}$ and

$$
G(x):=\left\{\frac{|\nabla v|^{2}}{1-v^{2}}-(A+b \sqrt{-K} \cos \theta)^{2}\right\} \cos ^{2} \theta .
$$


If $x_{0}$ is an interior point of $M$, the proof has been given above. Now we assume that $x_{0} \in \partial M$. Let $v_{\nabla u}$ be the unit normal vector that points outward $M$ with respect to $g_{\nabla u}$. Then

$$
D G\left(v_{\nabla u}\right)\left(x_{0}\right) \geq 0 \text {. }
$$

Since Neumann boundary condition yields $\nabla u \in T(\partial M)$, we have $D u\left(v_{\nabla u}\right)=$ $g_{\nabla u}\left(v_{\nabla u}, \nabla u\right)=0$. Noting that $\partial M$ is convex and the fact that $\nabla v=$ $\frac{2}{(1+k)(1+\varepsilon)} \nabla u$, one gets

$$
\begin{aligned}
D G\left(v_{\nabla u}\right) & =D g_{\nabla u}(\nabla v, \nabla v)\left(v_{\nabla u}\right)=2 g_{\nabla u}\left(D_{v_{\nabla u}}^{\nabla u} \nabla v, \nabla v\right) \\
& =2 g_{\nabla v}\left(v_{\nabla v}, D_{\nabla v}^{\nabla v} \nabla v\right) \leq 0
\end{aligned}
$$

at $x_{0}$. The last inequality follows from Lemma 3.1 and Lemma 3.2 in [19], which shows that it is equivalent to $g_{v}\left(v, D_{\nabla v}^{\nabla v} \nabla v\right) \leq 0$, where $v$ denotes the unit normal vector that points outwards $M$. The tangent derivative of $G$ obviously vanishes due to its maximality. Therefore

$$
\nabla^{\nabla u} G\left(x_{0}\right)=0 \text {. }
$$

The rest of the proof proceeds as the case that $\partial M=\emptyset$.

\section{The first Dirichlet eigenvalue}

Recall that under the weighted Ricci curvature conditions, the lower bound of the first eigenvalue on closed Finsler manifolds was obtained in [25], where the Obata type rigidity theorem was also established. In this section, we give the lower bound of the first Dirichlet (resp. Neumann) eigenvalue on compact Finsler manifolds.

THEOREM 3.1. Let $(M, F, d \mu)$ be a compact reversible Finsler n-manifold with a mean convex boundary. If $S$ curvature vanishes and the Ricci curvature satisfies Ric $\geq(n-1) k>0$, then the first Dirichlet eigenvalue of Finsler-Laplacian

$$
\lambda_{1} \geq n k \text {. }
$$

Proof. Let $u \in C_{0}^{\infty}(M)$ be a nonnegative function. By using Lemma 1.3 and the condition of Theorem 3.1, we have

$$
\begin{aligned}
\frac{1}{2} \Delta^{\nabla u}\left(|\nabla u|^{2}\right) & =D(\Delta u)(\nabla u)+|\nabla u|^{2} \operatorname{Ric}_{\infty}(\nabla u)+\left|\nabla^{2} u\right|_{H S(\nabla u)}^{2} \\
& \geq|\nabla u|^{2} \operatorname{Ric}_{\infty}(\nabla u)+D(\Delta u)(\nabla u)+\sum_{a=1}^{n} u_{a a}^{2} \\
& \geq|\nabla u|^{2} \operatorname{Ric}_{\infty}(\nabla u)+D(\Delta u)(\nabla u)+\frac{1}{n}\left(\sum_{a=1}^{n} u_{a a}\right)^{2}
\end{aligned}
$$




$$
\begin{aligned}
& =|\nabla u|^{2} \operatorname{Ric}(\nabla u)+D(\Delta u)(\nabla u)+\frac{(\Delta u)^{2}}{n} \\
& \geq(n-1) k|\nabla u|^{2}+D(\Delta u)(\nabla u)+\frac{(\Delta u)^{2}}{n} .
\end{aligned}
$$

Integrating both sides of the formula above and using the divergence theorem, we have

$$
\frac{1}{2} \int_{\partial M} g_{v}\left(v, \nabla^{\nabla u}|\nabla u|^{2}\right) d \zeta \geq \int_{M}\left[(n-1) k|\nabla u|^{2}+D(\Delta u)(\nabla u)+\frac{(\Delta u)^{2}}{n}\right] d \mu .
$$

where $d \zeta=v\rfloor d \mu$ denotes the induced volume form on $\partial M$ and $v$ denotes the unit normal vector outwards $\partial M$.

In what follows, we estimate $g_{v}\left(v, \nabla^{\nabla u}|\nabla u|^{2}\right)$ on $\partial M$. If $|\nabla u|\left(x_{0}\right)=0$, $x_{0} \in \partial M$, we draw geodesic $\gamma$ such that $\gamma(0)=x_{0}, \dot{\gamma}(0)=w$ for $w \in T_{x_{0}} M$. Then

$$
\begin{aligned}
\frac{\partial|\nabla u|^{2}}{\partial w}\left(x_{0}\right) & =\lim _{\gamma \ni x \rightarrow x_{0}} \frac{|\nabla u|^{2}(x)-|\nabla u|^{2}\left(x_{0}\right)}{d\left(x, x_{0}\right)} \\
& =\lim _{\gamma \ni x \rightarrow x_{0}} \frac{|\nabla u|^{2}(x)}{d\left(x, x_{0}\right)} \\
& =\lim _{\gamma \ni x \rightarrow x_{0}}|\nabla u|(x) \lim _{\gamma \ni x \rightarrow x_{0}} \frac{|\nabla u|(x)}{d\left(x, x_{0}\right)}=0 .
\end{aligned}
$$

By arbitrariness of $w$, we have $\nabla^{\nabla u}|\nabla u|^{2}=0$. If $x_{0} \in M_{u} \cap \partial M$, we can choose $\left\{e_{1}, \ldots, e_{n-1}, e_{n}=\frac{\nabla u}{|\nabla u|}\right\}$ as $g_{\nabla u}$-orthonormal basis. Then $v=-\frac{\nabla u}{|\nabla u|}$ is the unit normal vector outwards $\partial M$. Therefore, by Lemma 1.4, Definition 1.2 and the condition $S \equiv 0$, we get

$$
\begin{aligned}
g_{v}\left(v, \nabla^{\nabla u}|\nabla u|^{2}\right) & =-\frac{\nabla u\left(g_{\nabla u}(\nabla u, \nabla u)\right)}{|\nabla u|}=-2|\nabla u| u_{n n} \\
& =2|\nabla u|\left(-\Delta u+\sum_{a=1}^{n-1} u_{a a}-S(\nabla u)\right) \\
& =2|\nabla u|^{2} H-2|\nabla u| \Delta u \leq-2|\nabla u| \Delta u .
\end{aligned}
$$

From (3.1) we have

$$
-\int_{\partial M \cap M_{u}}|\nabla u| \Delta u d \zeta \geq \int_{M}\left[(n-1) k|\nabla u|^{2}+D(\Delta u)(\nabla u)+\frac{(\Delta u)^{2}}{n}\right] d \mu .
$$

By a classical density argument, the above formula still holds for the first Dirichlet eigenfunctions. In the following, we may as well assume $u$ is a first Dirichlet 
NEW LOWER BOUNDS OF THE FIRST EIGENVALUE ON FINSLER MANIFOLDS 337 eigenfunction relative to $\lambda_{1}$. Namely,

$$
\begin{cases}\Delta u=-\lambda_{1} u, & \text { in } M, \\ u=0, & \text { on } \partial M .\end{cases}
$$

Since $(M, F, d \mu)$ is a compact reversible Finsler $n$-manifold, we can suppose that $u>0$ in $M$ (see [6] for details). Notice that

$$
(\Delta u)^{2}=-\lambda_{1} u \Delta u=\lambda_{1}\left(|\nabla u|^{2}-\frac{1}{2} \Delta^{\nabla u} u^{2}\right) .
$$

Integrating both sides of (3.3) and using the divergence theorem and $\left.u\right|_{\partial M}=0$, one obtains

$$
\int_{M}(\Delta u)^{2} d \mu=\lambda_{1} \int_{M}|\nabla u|^{2} d \mu-\lambda_{1} \int_{\partial M} u g_{v}(v, \nabla u) d \zeta=\lambda_{1} \int_{M}|\nabla u|^{2} d \mu
$$

Combining it with (3.2) and noting $\left.\Delta u\right|_{\partial M}=0$, we get

$$
0 \geq \int_{M}\left[(n-1) k-\frac{n-1}{n} \lambda_{1}\right]|\nabla u|^{2} d \mu
$$

which implies $\lambda_{1} \geq n k$.

In what follows, we give the following result which can be obtained by a bit modification in the proof above.

Proposition 3.2. Let $(M, F, d \mu)$ be a compact Finsler n-manifold with a convex boundary. If the weighted Ricci curvature satisfies $\operatorname{Ric}_{N} \geq(n-1) k>0$ for $N \in(n, \infty)$, then the first Neumann eigenvalue of Finsler-Laplacian

$$
\lambda_{1} \geq \frac{n-1}{N-1} N k
$$

Proof. By a similar argument as Theorem 3.1, and using the relationship $\operatorname{Ric}_{N}=\operatorname{Ric}_{\infty}-\frac{S^{2}}{(N-n) F^{2}}$ and inequality $\frac{(a+b)^{2}}{n} \geq \frac{a^{2}}{N}-\frac{b^{2}}{N-n}$ for any $N \in$
$(n, \infty)$, we get

$$
\frac{1}{2} \int_{\partial M \cap M_{u}} g_{v}\left(v, \nabla \nabla|\nabla u|^{2}\right) d \zeta \geq \int_{M}\left[(n-1) k|\nabla u|^{2}+D(\Delta u)(\nabla u)+\frac{(\Delta u)^{2}}{N}\right] d \mu
$$

Since Neumann boundary condition gives $\nabla u \in T(\partial M)$, we have

$$
\int_{M}(\Delta u)^{2} d \mu=\lambda_{1} \int_{M}|\nabla u|^{2} d \mu-\lambda_{1} \int_{\partial M} u g_{v}(v, \nabla u) d \zeta=\lambda_{1} \int_{M}|\nabla u|^{2} d \mu .
$$

Therefore,

$$
\frac{1}{2} \int_{\partial M \cap M_{u}} g_{v}\left(v, \nabla^{\nabla u}|\nabla u|^{2}\right) d \zeta \geq \int_{M}\left[(n-1) k-\frac{N-1}{N} \lambda_{1}\right]|\nabla u|^{2} d \mu
$$


Next we have only to prove $g_{v}\left(v, \nabla^{\nabla u}|\nabla u|^{2}\right) \leq 0$ on $\partial M \cap M_{u}$ for the Neumann condition. In this case (see [19])

$$
g_{v}\left(v, \nabla^{\nabla u}|\nabla u|^{2}\right) \leq 0 \Leftrightarrow g_{\nabla u}\left(v_{\nabla u}, \nabla^{\nabla u}|\nabla u|^{2}\right) \leq 0 .
$$

Here $v_{\nabla u}$ denotes the unit outward normal vector with respect to $g_{\nabla u}$. Since $\partial M$ is convex, $g_{v}\left(v, D_{\nabla u}^{\nabla u} \nabla u\right) \leq 0$. Hence,

$$
\begin{aligned}
g_{\nabla u}\left(v_{\nabla u}, \nabla^{\nabla u}|\nabla u|^{2}\right) & =D g_{\nabla u}(\nabla u, \nabla u)\left(v_{\nabla u}\right) \\
& =2 g_{\nabla u}\left(D_{v_{\nabla u}}^{\nabla u} \nabla u, \nabla u\right)=2 g_{\nabla u}\left(v_{\nabla u}, D_{\nabla u}^{\nabla u} \nabla u\right) \\
& \leq 0 .
\end{aligned}
$$

The last inequality is due to Lemma 3.1 and Lemma 3.2 in [19], which shows that it is equivalent to $g_{v}\left(v, D_{\nabla u}^{\nabla u} \nabla u\right) \leq 0$.

\section{REFERENCES}

[1] D. BAKRY AND Z. M. QIAN, Some new results on eigenvectors via dimension, diameter, and Ricci curvature, Adv. Math. 155 (2000), 98-153.

[2] B. CHEN, Some geometric and analysis problems in Finsler geometry, report of postdoctoral research, Zhejiang University, 2010 (in Chinese).

[ 3 ] Y. W. Chu AND Z. J. Hu, Lower bound estimates of the first eigenvalue for the $f$-Laplacian and their applications, Quart. J. Math. 64 (2013), 1023-1041.

[4] M. F. Chen AND F. Y. WANG, General formula for lower bound of the first eigenvalue on Riemannian manifolds, Sci. China, Ser. A. 40 (1997), 384-394.

[ 5 ] A. Futaki, H. Z. Li And X. D. Li, On the first eigenvalue of the Witten-Laplacian and the diameter of compact shrinking Ricci solitons, Ann. Glob. Ana. Geom. 44 (2013), 105-114.

[6] Y. X. Ge AND Z. M. SHEN, Eigenvalues and eigenfunctions of metric measure manifolds, Proc. London Math. Soc. 82 (2001), 725-746.

[ 7 ] F. B. Hang AND X. D. WANG, A remark on Zhong-Yang's eigenvalue estimate, Int. Math. Res. Notices 2007 (2007), rnm064, doi:10.1093/imrn/rnm064.

[8] P. Li and S. T. YaU, Estimates of eigenvalues of a compact Riemannian manifold, Amer. Math. Soc. Proc. Sympos. Pure Math. 36 (1980), 205-239.

[9] A. Lichnerowicz, Geometries des groupes des transformations, Dunod, Paris, 1958.

[10] J. LING, Lower bounds of the eigenvalues of compact manifolds with positive Ricci curvature, Ann. Global Anal. Geom. 31 (2007), 385-408.

[11] J. LiNG, An exact solution to an equation and the first eigenvalue of a compact manifold, Illinois J. Math. 51 (2007), 853-860.

[12] J. Lott and C. Villani, Weak curvature bounds and functional inequalities, J. Funct. Anal. 245 (2007), 311-333.

[13] S. Онта, Finsler interpolation inequalities, Calc. Var. Partial Differential Equations 36 (2009), 211-249.

[14] S. Ohta And K.-T. Sturm, Bochner-Weitzenbock formula and Li-Yau estimates on Finsler manifolds, Adv. Math. 252 (2014), 429-448.

[15] Z. M. Qian, H. C. Zhang AND X. P. Zhu, Sharp spectral gap and Li-Yau's estimate on Alexandrov spaces, Math. Z. 273 (2013), 1175-1195.

[16] R. ReILly, Applications of the Hessian operatior in a Riemannian manifold, Indiana Univ. Math. J. 26 (1977), 459-472. 
[17] Z. M. SHEN, Lectures on Finsler geometry, World Scientific Publishing Co., Singapore, 2001.

[18] Z. M. SHEN, The non-linear Laplacian for Finsler manifolds, The theory of Finslerian Laplacians and applications (P. Antonelli, ed.), Proc. conf. on Finsler Laplacians, Kluwer Acad. Press, Netherlands, 1998.

[19] G. F. WANG AND C. XIA, A sharp lower bound for the first eigenvalue on Finsler manifolds, Ann. Inst. H. Poincaré Anal. Non Linéaire 30 (2013), 983-996.

[20] G. F. Wei and W. Wylie, Comparison geometry for the smooth metric measure spaces, Proceedings of the 4th International Congress of Chinese Mathematicians 2 (2007), 191-202.

[21] B. Y. WU AND Y. L. XIN, Comparison theorems in Finsler geometry and their applications, Math. Ann. 337 (2007), 177-196.

[22] H. C. YANG, Estimate for the first eigenvalue for a compact Riemannian manifold, Sci. China, Ser. A. 33 (1990), 39-51.

[23] D. G. YANG, Lower bound estimates on the first eigenvalue for compact manifolds with positive Ricci curvature, Pacific J. Math. 190 (1999), 383-398.

[24] S. T. Yin AND Q. He, Eigenvalue comparison theorems on Finsler manifolds, Chin. Ann. Math. 36B (2015), 31-44.

[25] S. T. Yin, Q. He AND Y. B. Shen, On lower bounds of the first eigenvalue of FinslerLaplacian, Publ. Math. Debrecen 83 (2013), 385-405.

[26] S. T. Yin, Q. He AND Y. B. Shen, On the first eigenvalues of Finsler manifolds with nonnegative weighted Ricci curvature, Sci. China Math. 57 (2014), 1057-1070.

[27] J. Q. ZhONG AND H. C. YANG, On the estimate of the first eigenvalue of a compact Riemannian manifold, Sci. China, Ser. A. 27 (1984), 1265-1273.

Song-Ting Yin

Department of Mathematics and Computer Science

TONGLING UNIVERSITY

Tongling, 244000 ANHuI

P.R. CHINA

E-mail: yst419@163.com

Qun $\mathrm{He}$

Department of Mathematics

TONGJI UNIVERSITY

ShanghaI, 200092

P.R. CHINA

E-mail: hequn@mail.tongji.edu.cn

Da-Xiao Zheng

Department of Mathematics

TONGJI UNIVERSITY

ShaNGHAI, 200092

P.R. CHINA

E-mail: 18917240898@163.com 